\title{
Effect of Erythropoiesis Stimulating Agents on Clinical Outcomes in Breast Cancer Patients: A Systematic Review of Randomised Controlled Trials
}

Saeeda Sabir1, Yusra Habib Khan², Maryam Khatoon', Rabia Noreen1, Tauqeer Hussain Mallhi2 and Nayyab Jabeen³

\author{
IInstitute of Pharmacy, Lahore College for Women University, Lahore, Pakistan \\ ${ }^{2}$ Department of Clinical Pharmacy, Jouf University, Al-Jouf, Kingdom of Saudi Arabia \\ 3Department of Medicine, Khalifa Gul Nawaz Teaching Hospital, MTI, Bannu, Khyber Pakhtunkhwa, Pakistan
}

\begin{abstract}
The impact of erythropoiesis stimulating agents (ESAs) on clinical outcomes among breast cancer patients is debatable. Current review is aimed to ascertain the efficacy of ESAs among breast cancer patients. Randomised controlled trials (RCTs) were electronically searched. Primary outcomes were mortality, blood transfusion requirements and thromboembolic events (TEEs); whereas, secondary outcomes were safety, tumor progression, anemia treatment, hemoglobin levels and quality of life (QOL). Out of 11 RCTs including 6,849 participants, 9 RCTs reported 2,312 deaths with overall mortality of $33.7 \%$. Mortality reported for epoetin alfa (EA), epoetin beta (EB) and darbepoetin alfa (DA) was $41.24 \%$, $73.1 \%$ and $8.99 \%$ respectively. TEEs reported for EA, EB and DA were $5.88 \%, 9.28 \%$ and $2.85 \%$, respectively. Serious adverse events were $39.04 \%, 36.29 \%, 1.53 \%$ for EA, EB and DA, respectively. Tumor progression for EA and EB was $37.53 \%$ and $95.46 \%$, respectively. No tumor progression was reported with DA. Erythropoietin reported no mortality, TEEs, serious ADRs and tumor progression. About $9 \%$ patients required transfusions during ESA therapy. Current evidence suggests that use of ESA reduces transfusion need but increases mortality and risks of TEEs.
\end{abstract}

Key Words: Chemotherapy, Randomised controlled trials, Anemia, Breast cancer, Erythropoiesis stimulating agents, Mortality, Tumor progression, Survival, Quality of life, Transfusion requirements, Safety.

How to cite this article: Sabir S, Khatoon M, Noreen, Mallhi TH, Khan YH, Jabeen N. Effect of erythropoiesis stimulating agents on clinical outcomes in breast cancer patients: A systematic review of randomised controlled trials. J Coll Physicians Surg Pak 2020; 30(3):292-298.

\section{INTRODUCTION}

Anemia is one of the major problems in patients receiving cancer chemotherapy for which blood transfusions or erythropoietin stimulating agents (ESAs) are considered. ESAs have demonstrated promising roles in decreasing transfusion requirement, improving hemoglobin levels and quality of life (QOL) in chemotherapyinduced anemia (CIA) among various types of cancer. 1-7 $\mathrm{CIA}$ in breast cancers (BC) has been corrected with the use of ESAs. However, contradictory results have surfaced about numerous clinical outcomes of ESAs such as increased mortality, tumor progression and increased number of thromboembolic events.8-18 The use of ESAs is still debatable and researchers are trying to evaluate their benefits which can outweigh the risks.

Though various trials have evaluated the efficacy of ESAs in $\mathrm{BC}$ patients, ${ }^{8-18}$ but composite evidence regarding the

Correspondence to: Dr. Yusra Habib Khan, Department of Clinical Pharmacy, College of Pharmacy, Jouf University, Al-Jouf, Kingdom of Sauid Arabia,

E-mail: yusrahabib@ymail.com

Received: February 06, 2019; Revised: June 24, 2019; Accepted: July 12, 2019 use of ESAs in BC is limited. A recent review investigated the impact of ESAs in managing cognitive alterations among BC patients. ${ }^{19}$ Another review concentrated on assessing the benefits of epoetin therapy among $\mathrm{BC}$ patients undergoing chemotherapy. ${ }^{20}$ Aapro et al., conducted a pooled analysis of 9 RCTs to investigate the efficacy and safety of ESAs in BC patients. ${ }^{21}$ However, there are few trials which were not incorporated in their study due to predetermined criteria of quantitative analysis. In this context, current systematic review was performed to accentuate the effects of ESAs for BC patients by including all available trials.

\section{METHODOLOGY}

This systematic review complies with the PRISMA statement. ${ }^{22}$ Cochrane Library, Google Scholar, Elsevier and PubMed were systematically searched for RCTs from 1989 to 2018. Search terms were chemotherapy, randomised controlled trials, anemia, breast cancer, erythropoiesis stimulating agents, erythropoietin, epoetin, darbepoetin, methoxy polyethylene glycolepoetin beta, mortality, tumor progression, survival, quality of life, transfusion requirements and safety.

Titles and abstracts were independently assessed by two authors (SS and RN). The inclusion of study in the 
review was based on full-text assessment. Dissent among researchers concerning the worth of studies was resolved through discussion and mutual consent. All RCTs conveying the effect of ESAs in BC were included. Studies conducted on other types of cancers, published in language other than English and having ambiguous inclusion were excluded. Primary outcome measures were mortality, blood transfusion requirements and thromboembolic events (TEEs). Secondary outcome measures were safety, tumor progression, anemia treatment, hemoglobin levels and quality of life (QOL).

Employing a pre-structured data collection form (DCF), data were independently extracted by two authors (MK \& FN). All the studies were evaluated to determine the effect of ESAs on predefined clinical outcomes (Figure 1).

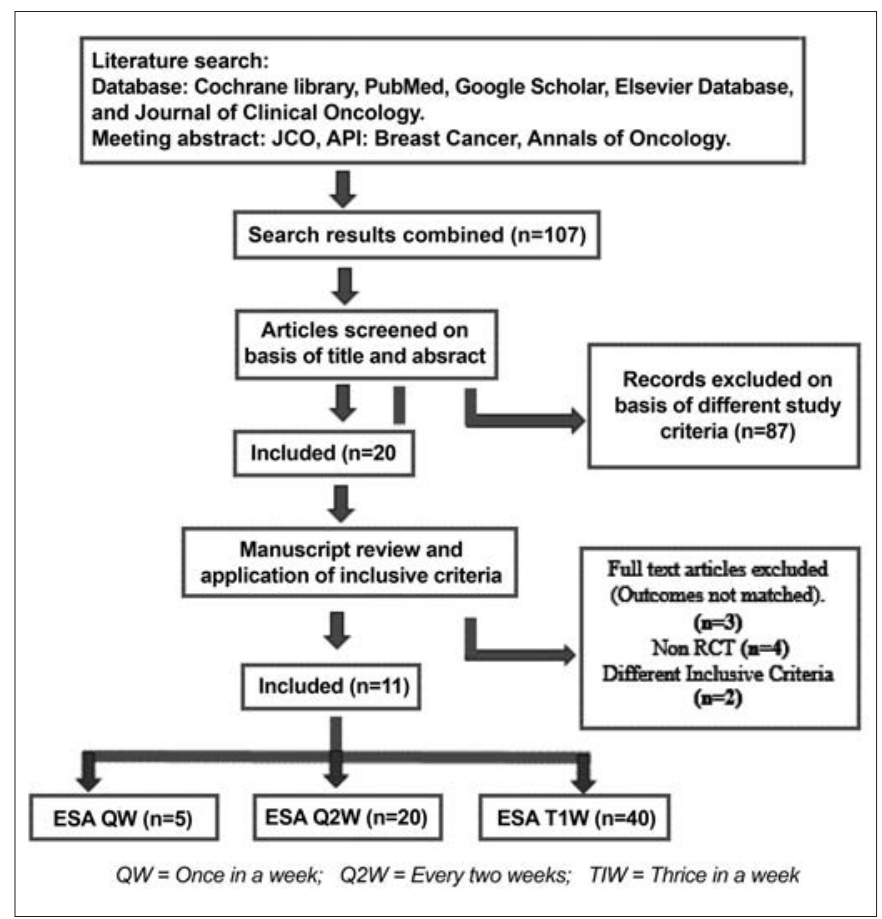

Figure 1: Schematic diagram representing the assortments of studies.

\section{RESULTS}

Characteristics of selected studies:

Eleven RCTs reporting the predetermined outcomes with the use of ESAs were included in current review.

The risk of bias within studies was assessed on the PRISMA criteria (Figure 2). All RCTs were adequately randomised, and appropriately concealed; most trials gave follow-up status up to last extent. Blinding of patients was done in only three trials.9,11,17 One trial stopped early for benefits and all the studies followed the intent to treat principle. ${ }^{11}$

RCTs were conducted on $6,849 \mathrm{BC}$ patients with CIA of age $\geq 18$ years (Table I). Four trials were conducted in Germany, ${ }^{14-17}$ four were located in multiple countries ${ }^{11-13,18}$ and the remaining three were from Italy, USA and Canada each. $8-10$

The interventions were ESAs in varying doses and frequencies with chemotherapy. Five RCTs had once weekly dosing, 9-12,18 two had twice weekly dosing 14,15 and four RCTs had thrice weekly dosing. $8,13,16,17$

All trials had at least one predefined outcome measure. Timings of outcome measures varied with different followup duration in these trials.

\section{Impact of ESAs on clinical outcomes:}

Nine trials reported mortality ranging from $1.9 \%$ to $73.1 \%$ in interventional group (IG) and $6 \%$ to $72.8 \%$ in control group (CG), suggesting the higher mortality in IG. However, only one study (Aapro et al. ${ }^{12}$ ) reported

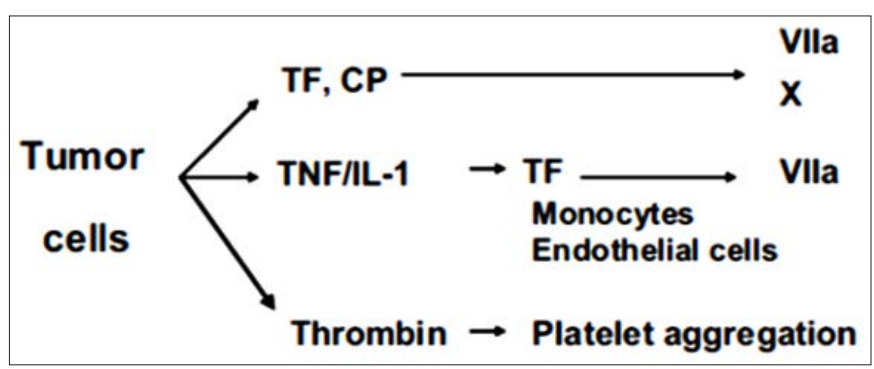

Figure 3: Pathways of activation of coagulation system in cancer patients. ${ }^{32}$ $C P=$ Cancer procoagulant; $I L-1=$ Interleukin-1; TF=Tissue factor; TNF=Tumor necrosis factor.

\begin{tabular}{|c|c|c|c|c|c|c|c|}
\hline Trials & $\begin{array}{l}\text { Concealment of } \\
\text { randomization }\end{array}$ & $\begin{array}{l}\text { RCT stopped } \\
\text { early }\end{array}$ & Patients blinded & HCP blinded & DC blinded & OA blinded & $\begin{array}{l}\text { Proportion of } \\
\text { patients lost } \\
\text { to follow-up }\end{array}$ \\
\hline Del Mastro et al. (1997) & $\checkmark$ & $x$ & NA & NA & NA & NA & 22 \\
\hline O'Shaughnessy et al. (2005) & $\checkmark$ & $x$ & $\checkmark$ & $\checkmark$ & NA & NA & 22 \\
\hline Chang et al. (2005) & $\checkmark$ & $x$ & $x$ & $x$ & NA & NA & 4 \\
\hline Jones et al. (2008) & $\checkmark$ & $\checkmark$ & $\checkmark$ & $\checkmark$ & $\checkmark$ & $\checkmark$ & 221 \\
\hline Aapro et al. (2008) & $\checkmark$ & $x$ & $x$ & $x$ & $x$ & $x$ & 123 \\
\hline Pronzato et al. (2010) & $\checkmark$ & $x$ & $x$ & $x$ & $\checkmark$ & $\checkmark$ & NA \\
\hline Untch et al. (2011) & $\checkmark$ & $x$ & $x$ & $x$ & $\checkmark$ & $\checkmark$ & NA \\
\hline Untch et al. (2011) & $\checkmark$ & $x$ & $x$ & $x$ & $\checkmark$ & $\checkmark$ & \\
\hline Moebus et al. (2013) & $\checkmark$ & $x$ & NA & NA & NA & NA & 70 \\
\hline Nitz et al. (2014) & $\checkmark$ & $x$ & $\checkmark$ & $\checkmark$ & $\checkmark$ & $\checkmark$ & NA \\
\hline Jones et al. (2016) & $\checkmark$ & $x$ & $x$ & $x$ & $x$ & $x$ & \\
\hline
\end{tabular}

$H C P=$ Health care provider; $D C=$ Data collectors, $O A=$ Outcome assessors.

Figure 2: Quality assessment of trials included in the review. 
Table I: Summary of included studies evaluating effects of ESA on various outcomes in breast cancer patients.

\begin{tabular}{|c|c|c|c|c|c|c|c|c|c|c|c|c|}
\hline Authors & $\mathrm{N}$ & IG & CG & Age & \begin{tabular}{|l|}
$\mathrm{BC}$ \\
stage
\end{tabular} & $\begin{array}{l}\text { Total } \\
\text { duration }\end{array}$ & Dose & Frequency & Dose & Frequency & Co-intervention & $\mathrm{F}$ \\
\hline Del Mastro et al., 19978 & 62 & 31 & 31 & $29-68$ & "11 & 28 months & $\begin{array}{l}\text { EPO - } 150 \mathrm{U} / \mathrm{kg}+3 \\
\text { MC same as CG }\end{array}$ & $\begin{array}{l}3 \text { times weekly } \\
\mathrm{E}-60 \mathrm{mg} / \mathrm{m}^{2}\end{array}$ & $\begin{array}{l}\mathrm{C}-600 \mathrm{mg} / \mathrm{m}^{2}, \\
\mathrm{E}-60 \mathrm{MG} / \mathrm{m}^{2} \\
\mathrm{~F}-600 \mathrm{mg} / \mathrm{m}^{2} \\
\text { (CEF) IV on day } 1 \\
\mathrm{G}-\mathrm{CSF}, 5 \mathrm{sug} / \mathrm{kg} \\
\text { subcutaneously from } \\
\text { day } 4 \text { to day } 11\end{array}$ & Every 2 weeks & $\begin{array}{l}\text { Ferrous sulfate } \\
325 \mathrm{mg} / \mathrm{d} \text { in specific } \\
\text { cases }\end{array}$ & 6 months \\
\hline O'Shaughnessy et al., $2005^{9}$ & 100 & 51 & 49 & $>18$ & $|-1|||$ & 10 months & $\begin{array}{l}\text { EA - 40,000 U, } \\
\text { increased to 60,000 U } \\
\text { if } \mathrm{Hb} \text { level did not } \\
\text { improve + } \\
\text { MC same as CG }\end{array}$ & Once weekly & Placebo + MC & Once weekly & . & Monthly \\
\hline Chang et al., $2005^{10}$ & 354 & 177 & 177 & $>18$ & I-IV & 7 months & $E A-40,000 U+M C$ & Once weekly & $\mathrm{SC}+\mathrm{MC}$ & & $200 \mathrm{mg} /$ day oral iron & Weekly \\
\hline Jones et al., 200511 & 939 & 469 & 470 & $\geq 18$ & I-IV & 12 months & EA - 40,000 U + & Once weekly & Placebo for 12 months + & Once weekly & & 3 months \\
\hline Aapro et al., $2008^{12}$ & 463 & 231 & 232 & $\geq 18$ & & 43 months & $\begin{array}{l}\text { EB - } 30,000 \mathrm{U}+ \\
\text { MC same as CG }\end{array}$ & Once weekly & $B S C+A / T B C$ & $\begin{array}{l}\text { Iron } \\
\text { supplementation }\end{array}$ & & $\begin{array}{l}\text { Monthly } \\
\text { for } 6 \\
\text { months } \\
\text { then } 3 \\
\text { months }\end{array}$ \\
\hline Pronzato et al., 201013 & 223 & 110 & 113 & $>18$ & I-IV & 19 months & $\begin{array}{l}\text { EA- initiated at } \\
10,000 \mathrm{IU}(5000 \mathrm{IU} \\
\text { if patient weight } \\
<45 \mathrm{~kg})+ \text { MC same } \\
\text { as CG }\end{array}$ & 3 times weekly & $\mathrm{BSC}+\mathrm{MC}$ & & & $\begin{array}{l}6 \text { months } \\
\text { then } \\
\text { annually }\end{array}$ \\
\hline Untch et al., 201114 & 733 & 356 & 377 & $18-65$ & IIIV & 32 months & $\begin{array}{l}\text { DA- } 4.5 \mu \mathrm{\mu g} / \mathrm{kg} \text { body } \\
\text { weight + Chemotherapy } \\
\text { same as CG }\end{array}$ & & $\begin{array}{l}\text { E- } 90 \mathrm{mg} / \mathrm{m}^{2}+ \\
\text { C- } 600 \mathrm{mg} / \mathrm{m}^{2} \text { by } \\
\text { T-175 mg/ } \mathrm{m}^{2} \text { (EC-T), } \\
\text { OR } \\
\text { E-150 mg/ } \mathrm{m}^{2} \text { followed } \\
\text { by T- } 225 \mathrm{mg} / \mathrm{m}^{2} \\
\text { with PF }(5 \mu \mathrm{g} / \mathrm{kg} / \mathrm{d} \text {, } \\
\text { d3-d10) followed by } \\
\text { CMF (C- } 500 \mathrm{mg} / \mathrm{m}^{2} \\
\mathrm{M}-40 \mathrm{mg} / \mathrm{m}^{2}, \mathrm{~F}-600 \\
\left.\mathrm{mg} / \mathrm{m}^{2}\right) \text { on days } 1 \\
\text { and } 8\left(E_{\mathrm{dd}}-T_{\mathrm{dd}}-C M F\right) \text {. }\end{array}$ & $\begin{array}{l}\text { same as CG } \\
\text { q14d x } 3\end{array}$ & & Annually \\
\hline Untch et al., 2011b & 733 & 356 & 377 & $18-65$ & IIIV & 32 months & $\begin{array}{l}\text { DA- } 4.5 \mu \mathrm{g} / \mathrm{kg} \text { body } \\
\text { weight + Chemotherapy } \\
\text { same as CG }\end{array}$ & Q2W & $\begin{array}{l}\text { E - } 90 \mathrm{mg} / \mathrm{m}^{2}+C-600 \\
\mathrm{mg} / \mathrm{m}^{2} \text { followed by } \\
\text { T- } 175 \mathrm{mg} / \mathrm{m}^{2} \text { (EC-T), } \\
\text { OR } \\
\text { E- } 150 \mathrm{mg} / \mathrm{m}^{2} \text { followed } \\
\text { by T- } 225 \mathrm{mg} / \mathrm{m}^{2} \text { with } \\
\text { PF }(5 \mu \mathrm{\mu g} / \mathrm{kg} / \mathrm{d} \text { d d3-d10) } \\
\text { followed by CMF } \\
\text { (C- } 500 \mathrm{mg} / \mathrm{m}^{2} \mathrm{M}- \\
40 \mathrm{mg} / \mathrm{m}^{2} \text {, } \\
\left.\text { F- } 600 \mathrm{mg} / \mathrm{m}^{2}\right) \text { on } \\
\text { days } 1 \mathrm{and} 8 \\
\left(E_{\mathrm{dd}} \mathrm{T}_{\mathrm{dd}}-\mathrm{CMF}\right) \text {. }\end{array}$ & $\begin{array}{l}\mathrm{q} 21 \mathrm{~d} \times 4 \\
\mathrm{q} 14 \mathrm{~d} \times 3 \\
\end{array}$ & & Annually \\
\hline Moebus et al., 201316 & 643 & 324 & 319 & $18-65$ & $|-| I||$ & 53 months & $\begin{array}{l}\text { EA- } 150 \mathrm{IU} / \mathrm{kg}+ \\
\text { Chemotherapy Same } \\
\text { as CG }\end{array}$ & \begin{tabular}{|}
3 times weekly- \\
Started on Day 1 \\
upto Day 14 after
\end{tabular} & \begin{tabular}{|l|} 
IDD chemotherapy- \\
Sequential adminis- \\
tration of each of three \\
cycles of E-( $150 \mathrm{mg} / \mathrm{m}^{2}$ \\
intravenously as a \\
bolus infusion), \\
T- $\left(225 \mathrm{mg} / \mathrm{m}^{2}\right.$ \\
intravenously as a 3-hour \\
infusion), and \\
C-( $2500 \mathrm{mg} / \mathrm{m}^{2}$ \\
intravenously as a \\
2-hour infusion), \\
respectively. \\
All patients received \\
filgrastim SC \\
$(5 \mu \mathrm{g} / \mathrm{kg}$ body weight \\
per day) from days 3 \\
to 10 of each cycle. \\
\end{tabular} & every 2 weeks & $200 \mathrm{mg} /$ day oral iron & Annually \\
\hline Nitz et al., 201417 & 1,234 & 615 & 619 & $>18$ & & 53 months & 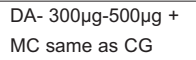 & 3 times weekly & $\mathrm{SC}+\mathrm{MC}$ & & & Annually \\
\hline Jones et al., 201618 & 2,098 & 1,050 & 1,048 & $>18$ & I-IV & 100 months & $\begin{array}{l}\text { EA-40,000IU + } \\
\text { MC same as CG }\end{array}$ & Once Weekly & $\mathrm{BSC}+\mathrm{MC}$ & & Oral \& IV iron therapy & \\
\hline
\end{tabular}

$I D D=$ Intense Dose Dense, E=Epirubicin, $T=P$ aclitaxel $C=C y c l o p h$. Based Chemotherapy, DA= Darbepoetin Alfa, IG = Intervention Group, CG = Control Group, BSC= Best Standard Care, SC= Standard Care, MC= Myelotoxic Chemotherapy, FLC= First - ine Chemotherapy, G-CSF = Granulocyte Colony-Stimulating Factor. 
statistically insignificant difference in mortality rate between both groups. Highest mortality rate was observed with EB. 12

Eight trials reported transfusion requirements for anemia ranging from $0 \%$ to $14.2 \%$ in IG and $0 \%$ to $28.1 \%$ in CG. Moebus et al. 16 reported considerably higher transfusion rate in CG $(28.1 \%)$ than IG (12.8\%). Need of transfusions was statistically higher in IG as compared to CG in six trials (Table II). Studies using DA reported lowest transfusion rate. 15

TEEs were reported in eight trials ranging from $2.8 \%$ to $16 \%$ in IG and $0.8 \%$ to $14 \%$ in CG, suggesting the higher events in IG. A statistically higher proportion of TEEs in IG were reported in five trials (Table II).

Nine studies reported adverse events (AEs) ranging from $0.28 \%$ to $57.6 \%$ in IG and $0.28 \%$ to $60 \%$ in CG. Serious AEs included extra-cardiac, erythrocyte, platelet, bleeding/clotting, gastric/duodenal, small/large bowel and mucous membrane disorders. Two studies indicated statistically higher AEs in IG as compared to CG, (Table III). ${ }^{11,17} \mathrm{DA}$ is associated with minimum number of ADEs in patients. ${ }^{15}$

Five trials reported tumor progression ranging from $41 \%$ to $95.2 \%$ in IG and $43 \%$ to $96 \%$ in CG, indicating the

Table II: RCTs evaluating effects of ESAs on mortality, transfusion requirements and thromboembolic events.

\begin{tabular}{|c|c|c|c|c|c|c|c|c|c|c|c|c|}
\hline \multirow{2}{*}{$\begin{array}{l}\text { Outcomes } \\
\text { Author, Year }\end{array}$} & \multicolumn{4}{|c|}{ Mortality } & \multicolumn{4}{|c|}{ Transfusion Requirements } & \multicolumn{4}{|c|}{ Thromboembolic Events } \\
\hline & Total & IG & CG & p-value & $\begin{array}{c}\text { Total } \\
\text { No. of } \\
\text { patients } \\
\text { receiving } \\
\text { transfusion }\end{array}$ & IG & CG & p-value & $\begin{array}{l}\text { Total } \\
\text { No. of } \\
\text { thrombo- } \\
\text { embolic } \\
\text { events }\end{array}$ & IG & CG & $p$-value \\
\hline Del Mastro et al., 19978 & & & & & 2 & $0 \%$ & $6.4 \%$ & & & & & \\
\hline O'Shaughnessy et al., $2005^{9}$ & 1 & $1.9 \%$ & & & & & & & & & & \\
\hline Chang et al., $2005^{10}$ & 51 & $13.5 \%$ & $15.2 \%$ & & 55 & $8.6 \%$ & $22.9 \%$ & $<0.0001$ & 33 & $10.8 \%$ & $7.8 \%$ & \\
\hline Jones et al., 200511 & 249 & $28 \%$ & $23 \%$ & 0.02 & 113 & $10 \%$ & $14 \%$ & 0.06 & 141 & $16 \%$ & $14 \%$ & \\
\hline Aapro et al., $2008^{12}$ & 338 & $73.1 \%$ & $72.8 \%$ & 0.522 & 96 & $14.2 \%$ & $27 \%$ & $? 0.001$ & 43 & $12.5 \%$ & $6 \%$ & 0.012 \\
\hline Pronzato et al., $2010^{13}$ & 43 & $20.9 \%$ & $17.7 \%$ & 0.86 & 26 & $7.5 \%$ & $16.5 \%$ & 0.059 & 5 & $3.6 \%$ & $0.8 \%$ & \\
\hline Untch et al., 201114 & 107 & $17 \%$ & $13 \%$ & 0.450 & & & & & & & & \\
\hline Untch et al., 201115 & & & & & 1 & $0.28 \%$ & $0 \%$ & - & 32 & $6 \%$ & $3 \%$ & 0.055 \\
\hline Moebus et al., $2013^{16}$ & 116 & $19 \%$ & $17 \%$ & & 131 & $12.8 \%$ & $28.1 \%$ & $<0.0001$ & 33 & $7 \%$ & $3 \%$ & 0.030 \\
\hline Jones et al., $2016^{18}$ & 1,337 & $64.8 \%$ & $63 \%$ & & 180 & $5.8 \%$ & $11.4 \%$ & $<0.001$ & 44 & $2.8 \%$ & $1.4 \%$ & 0.038 \\
\hline
\end{tabular}

IG=Intervention Group, $C G=$ Control Group

Table III: RCTs evaluating effects of ESAs on safety, tumor progression and hemoglobin levels.

\begin{tabular}{|c|c|c|c|c|c|c|c|c|c|c|c|c|}
\hline \multirow{2}{*}{$\begin{array}{l}\text { Outcomes } \\
\text { Author, Year }\end{array}$} & \multicolumn{4}{|c|}{ Safety } & \multicolumn{4}{|c|}{ Tumor progression } & \multicolumn{4}{|c|}{ Hemoglobin levels } \\
\hline & $\begin{array}{l}\text { Total } \\
\text { No. of } \\
\text { serious } \\
\text { AEs }\end{array}$ & IG & CG & p-value & \begin{tabular}{|c|} 
Total \\
No. of \\
cases of \\
tumor \\
progression
\end{tabular} & IG & CG & p-value & $\begin{array}{c}\text { Total } \\
\text { No. of } \\
\text { patients } \\
\text { with } \mathrm{Hb} \\
\text { change }\end{array}$ & IG & CG & $p$-value \\
\hline Del Mastro et al., 19978 & & & & & & & & & & No decline & Decline & $<0.001$ \\
\hline O'Shaughnessy et al., $2005^{9}$ & & & & & & & & & 93 & $\begin{array}{c}92.1 \% \\
\text { maintained }\end{array}$ & \begin{tabular}{c|}
$6.1 \%$ \\
maintained
\end{tabular} & \\
\hline Chang et al., $2005^{10}$ & \multicolumn{4}{|c|}{ Incidence similar between both groups } & & & & & 100 & $\begin{array}{c}51.4 \% \\
\text { maintained }\end{array}$ & \begin{tabular}{|c|}
$5.1 \%$ \\
maintained
\end{tabular} & $<0.0001$ \\
\hline Jones et al., 200511 & 357 & $42 \%$ & $34 \%$ & 0.02 & 394 & $41 \%$ & $43 \%$ & 0.98 & & $\begin{array}{c}59 \% \\
\text { maintained }\end{array}$ & \begin{tabular}{|c|}
$45 \%$ \\
maintained
\end{tabular} & $<0.001$ \\
\hline Aapro et al., $2008^{12}$ & 168 & $42 \%$ & $31 \%$ & & 442 & $95.2 \%$ & $96 \%$ & 0.448 & & $\begin{array}{c}68 \% \\
\text { maintained }\end{array}$ & \begin{tabular}{|c|}
$14 \%$ \\
maintained
\end{tabular} & $<0.001$ \\
\hline Pronzato et al., $2010^{13}$ & 34 & $16.5 \%$ & $14.4 \%$ & & \multicolumn{4}{|c|}{ No differences found } & & \begin{tabular}{c|}
$62 \%$ \\
maintained
\end{tabular} & \begin{tabular}{|c|}
$28 \%$ \\
maintained
\end{tabular} & $<0.001$ \\
\hline \multicolumn{13}{|l|}{ Untch et al., $20111^{14}$} \\
\hline Untch et al., $2011^{15}$ & 2 & $0.28 \%$ & $0.28 \%$ & & & & & & & $\begin{array}{c}\text { No } \\
\text { significant } \\
\text { change }\end{array}$ & $\begin{array}{c}\text { Significant } \\
\text { Decline }\end{array}$ & \\
\hline Moebus et al., $2013^{16}$ & 73 & $10 \%$ & $13 \%$ & & \multicolumn{4}{|c|}{ No deleterious effects } & & $\begin{array}{c}\text { No } \\
\text { decline }\end{array}$ & Declined & $<0.001$ \\
\hline Nitz et al., 201417 & 28 & $3.3 \%$ & $1.3 \%$ & 0.013 & & & & & & $\begin{array}{c}10.9 \% \\
\text { Declined }\end{array}$ & $\begin{array}{c}23.8 \% \\
\text { Declined }\end{array}$ & 0.025 \\
\hline Jones et al., $2016^{18}$ & 1,237 & $57.6 \%$ & $60 \%$ & & 1,241 & $58.1 \%$ & $60.1 \%$ & & & & & \\
\hline
\end{tabular}


Table IV: RCTs evaluating effects of ESAs on anemia treatment and quality of life.

\begin{tabular}{|c|c|c|c|c|c|c|c|c|}
\hline \multirow{2}{*}{$\frac{\text { Outcomes }}{\text { Author, Year }}$} & \multicolumn{4}{|c|}{ Anemia treatment } & \multicolumn{4}{|c|}{ Quality of life ${ }^{* *}$} \\
\hline & $\begin{array}{c}\text { Total No. of } \\
\text { patients treated }\end{array}$ & $\begin{array}{c}\text { IG } \\
\text { p-value }\end{array}$ & CG & p-value & $\begin{array}{c}\text { Total No. of } \\
\text { patients with } \\
\text { increased QOL }\end{array}$ & IG & CG & $\mathrm{p}$-value \\
\hline \multicolumn{9}{|l|}{ Del Mastro et al., 19978} \\
\hline O'Shaughnessy et al., $2005^{9}$ & & & & & 75 & $78.4 \%$ & $71.4 \%$ & \\
\hline Chang et al., $2005^{10}$ & 333 & $97.2 \%$ & $90.9 \%$ & - & 166 & $\begin{array}{c}93.8 \% \\
\text { change from } \\
\text { beseline }\end{array}$ & $\begin{array}{c}-95.4 \% \\
\text { change from } \\
\text { baseline }\end{array}$ & $<0.0001$ \\
\hline Jones et al., 200511 & - & - & - & - & \multicolumn{3}{|c|}{ No differences found } & 0.01 \\
\hline Aapro et al., 200812 & 359 & $82.6 \%$ & $72 \%$ & $\leq 0.01$ & \multicolumn{3}{|c|}{ No significant difference } & $>0.6$ \\
\hline Pronzato et al., $2010^{13}$ & - & $\begin{array}{c}14.2 \%{ }^{*} \\
\text { change from } \\
\text { baseline }\end{array}$ & $\begin{array}{c}-0.5 \%{ }^{*} \\
\text { change from } \\
\text { baseline }\end{array}$ & 0.002 & & $\begin{array}{c}18.6 \% \\
\text { change from } \\
\text { baseline }\end{array}$ & $\begin{array}{c}-2.7 \% \\
\text { change from } \\
\text { baseline }\end{array}$ & 0.003 \\
\hline \multicolumn{9}{|l|}{ Untch et al., 201114} \\
\hline Moebus et al., $2013^{16}$ & & & & & \multicolumn{4}{|c|}{ Not presented due to missing baseline data } \\
\hline Nitz et al., $2014^{17}$ & \multicolumn{4}{|c|}{ Not presented due to missing baseline data } & \multicolumn{4}{|c|}{ Not presented due to missing baseline data } \\
\hline Jones et al., $2016^{18}$ & 1,992 & $96 \%$ & $93.9 \%$ & & & & & \\
\hline
\end{tabular}

higher tumor progression in CG (Table III). EB is associated with maximum tumor progression rates ${ }^{12}$ as compared to EA. ${ }^{11}$

Nine trials reported hemoglobin $(\mathrm{Hb})$ change during ESA treatment. Of these, five trials reported that maintaining $\mathrm{Hb}$ was significant in IG ranging from $51.4 \%$ to $92.1 \%, 9-13$ $\mathrm{Hb}$ was maintained $5.1 \%$ to $45 \%$ patients in CG. Three trials reported significant $\mathrm{Hb}$ decline in CG.8,15,16 (Table IV). Maximum $\mathrm{Hb}$ maintenance is reported with EA. 9

Five out of 11 trials reported data on anemia treatment. Two trials used FACT-An Scale for scoring where Pronzato et al. ${ }^{13}$ reported $14.2 \%$ change of score in IG and $-0.5 \%$ in CG. Nitz et al. ${ }^{17}$ reported no significant difference of scores between IG and CG. Other three trials described percentage of patients with anemia free survival ranging from $82.6 \%$ to $97.2 \%$ in IG and $72 \%$ to $93.9 \%$ in CG (Table IV). Maximum rate of anemia treatment was associated EA. ${ }^{10,18}$

QOL was assessed in 7 RCTs. Of these, three trials demonstrated no impact on QOL with the use of ESAs. ${ }^{11,12,17}$ O'Shaughnessy et al. ${ }^{9}$ reported improvements in QOL in $78.4 \%$ in IG and $71.4 \%$ in CG using LASA scoring. Pronzato et al.13 reported results by CLAS Scale. Moebus et al. ${ }^{16}$ was unable to report QOL due to missing baseline data. All three scales provided statistically significant improvement in QOL in IG (Table IV). EA therapy showed maximum improvements in $\mathrm{QOL}$ score. 10

\section{DISCUSSION}

Anemia frequently occurs among cancer patients receiving chemotherapy. ${ }^{16}$ ESAs provide survival benefits from CIA in patients with BC. ${ }^{17}$ Anthracycline therapy as FEC combination including 5-fluorouracil with epirubicin, and cyclophosphamide induces anemia in almost $42 \%$ patients. ${ }^{16}$ Current review has analysed the impact of several ESAs on various outcomes among patients with $\mathrm{BC}$.

The high mortality rate after using ESAs is attributed to repopulating capability of tumor from a single stem cell in BC. Reinbothe et al. suggested that erythropoietin receptor $(E p o R)$ protein is expressed in breast tumor cells, where it seems to stimulate proliferation by erythropoietin-independent mechanism in estrogen receptor positive $(E R \alpha+)$, expressing in metastatic breast cancerous cells. ${ }^{23}$ Phillips et al. revealed that over expression of an erythropoietin receptor (EpoR) amplified the clonogenicity of cancer cells resulting in increased mortality after using ESAs. ${ }^{24}$ Abundant expression of c-Myc in many cancers is another reason of tumor progression with the use of ESAs. ${ }^{25}$ EPO also increases MYC expression in erythroid progenitor cells. MYC is a family of regulator genes as well as proto oncogenes that code for transcription factors. ${ }^{26}$

Previous studies revealed that treatment with epoetin alfa sustained and/or enhanced $\mathrm{Hb}$ concentration and patient reported outcomes (PROs). 10 Several quantitative analysis provides evidence of $\mathrm{Hb}$ elevation with the use of ESAs, thereby reducing the need of RBCs transfusion.7,10 Further clarification might include elevated oxygenation of tissue having tumor at greater $\mathrm{Hb}$ points. ${ }^{27}$ Cancer cells become unaffected by tumor hypoxic conditions, raised oxygenation inhibits hypoxia preserving tumor cells to be sensitive to radiation, and cytostatic therapy. Hypoxia is more prevalent in anemic patients. ${ }^{27}$ Epoetin was initially used as optional treatment therapy was for adjustment of anemia to avoid transfusions. ${ }^{28}$ European guidelines suggest the dose of erythropoietin as once weekly for patients having $\mathrm{Hb}$ between 9 to $11 \mathrm{~g} / \mathrm{dL}$ with target $\mathrm{Hb}$ of $12-13 \mathrm{~g} / \mathrm{dL} .12$ 
American guidelines recommends that erythropoietin should be reserved for patients having $\mathrm{Hb}<10 \mathrm{~g} / \mathrm{dL}$ to achieve targeted $\mathrm{Hb} 12 \mathrm{~g} / \mathrm{dL} .12$

Pronzato et al. estimated better tolerability of epoetin alfa with few AEs including thromboembolic events. ${ }^{13}$ Though occurrence of venous thrombosis was similar in both groups but serious thrombovascular events were more prevalent in IG receiving epoetin alfa. 13 The drugs for $B C$ have been connected with higher risk of venous TEEs due to thrombus formation in venous circulation. ${ }^{29}$ TEEs may reside due to both superficial venous thrombosis and deep venous thrombosis. ${ }^{30}$ TEEs can be determined primarily by the differences in underlying cancer population due to disease stage and activation of the coagulation system after using ESAs. ${ }^{31}$ Coagulation pathways in $B C$ are precised in Figure 2. ESAs can activate tumor cells to produce TF and cancer procoagulant $(\mathrm{CP})$, which can start the extrinsic pathway by activating certain coagulation factors (VIla and Xa). Thrombin causes platelet accumulation which intensifies the thrombophilic state. Hereafter, TF can initiate a hypercoagulability state with thrombosis. ${ }^{32}$

Since thrombotic events are second leading cause of death in cancer patients, thromboprophylaxis improves prognosis and QOL in BC patients by inhibiting the thrombotic events. ${ }^{33}$ However, Several regulatory authorities have limited the use of ESAs for CIA.16 Existing data underscored the association of epoetin and darbepoetin with TTEs and amplified mortality. 16 Similar findings have been reported in composite analysis of previous studies. ${ }^{21}$ However, epoetin alfa therapy caused substantial decrease in transfusion requirements as well as improvement in QOL. ${ }^{16}$

Del Mastro et al. reported that EPO prevents anemia and maintained $\mathrm{Hb}$ values along with prevention of anemia among receiving chemotherapy consisting of six cycles (cyclophosphamide, epirubicin, and fluorouracil (CEF) on day 1 , every two weeks using granulocyte colony stimulating factor, subcutaneously from day $4-11) .8$ The improved erythropoiesis with EPO therapy led to quick reduction of iron supply as evidenced by decline in iron/transferrin levels in plasma and overall iron stores assessed by total iron binding capacity. The boosted EPO-induced erythropoiesis is predictable to produce low level of ferritin. Representation of RBC is performed by estimation of mean corpuscular $\mathrm{Hb}(\mathrm{MCH})$, mean corpuscular volume (MCV), and mean corpuscular $\mathrm{Hb}$ concentration $(\mathrm{MCHC})$. Clinically, anemia does not occur in EPO group due to maintenance of $\mathrm{Hb}$ levels. ${ }^{8}$

\section{CONCLUSION}

Current review suggests that ESAs are generally well endured and can shield against anemia. With the exception of risks of thrombotic complications, ESAs appear to be harmless for the treatment or fundamental anticipation of anemia in CIA. Current evidence also ascertains that ESAs reduce the need for blood transfusions. However, risks of increased mortality and TEEs should not be disregarded during the treatment.

\section{CONFLICT OF INTEREST:}

Authors declared no conflict of interest.

\section{AUTHORS' CONTRIBUTION:}

YHK: Conceptualised the plan for the current study; assisted in interpreting the results; assessed the data quality and approved the final version of the manuscript for submission.

SS, THM, MK: Did the literature search, screening and data extraction.

RN, NJ: Performed critical appraisal of all included studies.

THM: Drafted the manuscript; assessed the data quality and approved the final version of the manuscript for submission.

All authors have critically reviewed the manuscript.

\section{REFERENCES}

1. Ludwig H, Fritz E, Kotzmann H. Erythropoietin treatment of anemia associated with multiple myeloma. N Engl J Med 1990; 322:1693-9.

2. Abels RI. Recombinant human erythropoietin in the treatment of the anemia of cancer. Acta Haemotol 1992; 87(suppl 1):4-11.

3. Leitgeb C, Pecherstorfer M, Fritz E. Quality of life in chronic anemia of cancer during treatment with recombinant human erythropoietin. Cancer 1994; 73:2535-42.

4. Glaspy J, Bukowski R, Steinberg D. For the procrit study group. Impact of therapy with epoetin alfa on clinical outcomes in patients with nonmyeloid malignanacies during cancer therapy in community oncology practice. J Clin Oncol 1997; 15:1218-34.

5. Demetri GD, Kris M, Wade J. Quality-of-life benefit in chemotherapy patients treated with epoetin alfa is independent of disease response or tumor type. Results from a prospective community oncology study. J Clin Oncol 1998; 16:3412-25.

6. Gabrilove JL, Cleeland CS, Livingston RB. Clinical evaluation of once-weekly dosing of epoetin alfa in chemotherapy patients: Improvements in hemoglobin quality of life. J Clin Oncol 2001; 19:2875-82.

7. Littlewood TJ, Bajetta E, Nortier JWR. Effects of epoetin alfa on hematologic paraments and quality of life in cancer patients receiving nonplatinum chemotherapy: Results of a randomized, double-blind, placebo-controlled trial. J Clin Oncol 2001; 19: 2865-74.

8. Del Mastro L, Venturini M, Lionetto R, Garrone O, Melioli G, Pasquetti $W$, et al. Randomized phase III trial evaluating the role of erythropoietin in the prevention of chemotherapyinduced anemia. J Clin Oncol 1997; 15:2715-21.

9. O'Shaughnessy JA, Vukelja SJ, Holmes FA. Feasibility of quantifying the effects of epoetin alfa therapy on cognitive function in women with breast cancer undergoing adjuvant or neoadjuvant chemotherapy. Clinical Breast Cancer 2005; 5: 439-46. 
10. Chang J, Couture F, Young S. Weekly epoetin alfa maintains hemoglobin, improves quality of life, and reduces transfusion in breast cancer patients receiving chemotherapy. J Clin Oncol 2005; 23:2597-2605.

11. Jones BL, Semiglazov V, Pawlicki M. Maintaining normal hemoglobin levels with epoetin alfa mainly in nonanemic patients with metastatic breast cancer receiving first line chemotherapy: A survival study. J Clin Oncol 2005; 23:5960-72.

12. Aapro M, Leonard RC, Barnadas A. Effect of once weekly epoetin beta on survival in patients with metastatic breast cancer receiving anthracycline-and/or taxane-based chemotherapy: Results of the breast cancer - anemia and the value of erythropoietin (BRAVE) study. J Clin Oncol 2008; 26:592-98.

13. Pronzato $P$, Cortesi $E$, Carin $C$. Epoetin alfa improves anemia and anemia-related, patient-reported outcomes in patients with breast cancer receiving myelotoxic chemotherapy: Results of a European, multicenter, randomized, controlled trial. Oncologist 2010; 15:935-43.

14. Untch M, Minckwitz G, Konecny GE. Prepare trial: A randomised phase III trial comparing preoperative, dose-dense, doseintensified, chemotherapy with epirubicin, paclitaxel, and CMF versus a standard-dose epirubicin-cyclophosphamide followed by paclitaxel with or without darbepoetin afla in primary breast cancer-outcome on prognosis. Ann Oncol 2011a; 22:1999-2006.

15. Untch M, Minckwitz G, Konecny GE. Prepare trial: A randomised phase III trial comparing preoperative, dosedense, dose-intensified, chemotherapy with epirubicin, paclitaxel, and CMF versus a standard- dose epirubicin/ cyclophosphamide followed by paclitaxel \pm darbepoetin afla in primary breast cancer-results at the time of surgery. Ann Oncol 2011b; 22:1988-98.

16. Moebus V, Jackisch $C$, Schneeweiss A. Adding epoetin alfa to intense dose-dense adjuvant chemotherapy for breast cancer: Randomized clinical trial. J Natl Cancer Inst 2013; 105:1018-26.

17. Nitz U, Gluz O, Zuna I. Final results from the prospective phase III WSG-ARA trial: Impact of adjuvant darbepoetin alfa on event-free survival in early breast cancer. Ann Oncoly 2014; 25:75-80.

18. Jones BL, Bondarenko I, Nemsadze G. A randomized, openlabel, multicenter, phase III study of epoetin alfa versus best standard of care in anemic patients with metastatic breast cancer receiving standard chemotherapy. J Clin Oncol 2016; 34:1197-207.

19. Chan RJ, McCarthy AL, Devenish J. Systematic review of pharmacologic and non-pharmacologic interventions to manage cognitive alterations after chemotherapy for breast cancer. Eur J Cancer 2015; 51:437-50.

20. Demetri GD, Gabrilove JL, Blasi MV. Benefits of epoetin alfa in anemic breast cancer patients receiving chemotherapy. Clin Breast Cancer 2002; 3:45-51.
21. Aapro M, Moebus V, Nitz U, O'Shaughnessy J, Pronzato P, Untch $\mathrm{M}$, et al. Safety and efficacy outcomes with erythropoiesis-stimulating agents in patients with breast cancer: $\mathrm{A}$ meta-analysis. Ann Oncol 2015, 26:688-95.

22. Moher D, Liberati A, Tetzlaff J, Altman DG. Preferred reporting items for systematic reviews and meta-analyses: The PRISMA statement. Ann Inter Med 2009; 151:264-9.

23. Reinbothe S, Larsson AM, Vaapil M, Wigerup C, Sun J, Jögi A. EPO-independent functional EPO receptor in breast cancer enhances estrogen receptor activity and promotes cell proliferation. Biochem Biophys Res Commun 2014; 445:163-9.

24. Phillips TM, Kim K, Vlashi E, McBride WH, Pajonk F. Effects of recombinant erythropoietin on breast cancer-initiating cells. Neoplasia 2007; 9:1122-9.

25. Ley R, Ewings KE, Hadfield K, Cook SJ. Regulatory phosphorylation of Bim: Sorting out the ERK from the JNK. Cell Death Differ 2005; 12:1008-14.

26. Adhikary S, Eilers M. Transcriptional regulation and transformation by Myc proteins. Nat Rev Molec Cell Biol 2005; 6: 635.

27. Bohlius J, Schmidlin K, Brillant C, Schwarzer G, Trelle S, Seidenfeld J, et al. Recombinant human erythropoiesisstimulating agents and mortality in patients with cancer: A meta-analysis of randomised trials. Lancet 2009; 373:1532-42.

28. Fishbane, S, Besarab A. Mechanism of increased mortality risk with erythropoietin treatment to higher hemoglobin targets. Clin J Am Society Nephrol 2007; 2:1274-82.

29. Rizzo JD, Brouwers M, Hurley P, Seidenfeld J, Arcasoy MO, Spivak JL, et al. American society of hematology / American society of clinical oncology clinical practice guidelines update on the use of epoetin and darbepoetin in adult patients with cancer. Blood 2010; 116:4045-59.

30. Timp JF, Braekkan SK, Versteeg HH, Cannegieter SC. Epidemiology of cancer-associated venous thrombosis. Blood 2013; 122:1712-23.

31. Aapro M, Scherhag A, Burger HU. Effect of treatment with epoetin- $\beta$ on survival, tumour progression and thromboembolic events in patients with cancer: An updated meta-analysis of 12 randomised controlled studies including 2301 patients. $\mathrm{Br} \mathrm{J}$ Cancer 2008; 99:14.

32. Fandrey J, Dicato M. Examining the involvement of erythropoiesis-stimulating agents in tumor proliferation (erythropoietin receptors, receptor binding, signal transduction), angiogenesis, and venous thromboembolic events. Oncologist 2009; 14(Supplement 1):34-42.

33. Khorana AA, Francis CW, Culakova E, Kuderer NM, Lyman GH. Thromboembolism is a leading cause of death in cancer patients receiving outpatient chemotherapy. J Thromb Haemost 2007; 5:632-4. 